\title{
Epoca reproductiva y talla media de primera madurez sexual de Strangomera bentincki y Engraulis ringens en el período 1993-1997, en la zona centro-sur de Chile
}

\author{
Luis Cubillos S., Mariella Canales A., Doris Bucarey S., Andrea Rojas S. y Rubén Alarcón M. \\ Instituto de Investigación Pesquera \\ Casilla 350, Talcahuano, Chile \\ E-mail: inpesca@arauco.reuna.cl
}

Recibido 17 diciembre 1998; versión corregida 20 septiembre 1999; aceptado 19 octubre 1999.

RESUMEN. Se analiza la época reproductiva de Strangomera bentincki y Engraulis ringens y se estima la talla promedio de primera madurez sexual $\left(\mathrm{Lm}_{50 \%}\right)$ para el período 1993-1997. Los indicadores reproductivos utilizados para analizar la época reproductiva son el índice gonadosomático (IGS) y la frecuencia temporal de los estados de

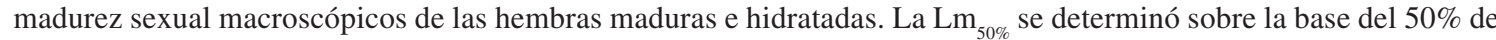
hembras maduras ajustando un modelo logístico. El mes en que ocurre el máximo IGS, en el período de mayor actividad reproductiva que se extiende entre julio y septiembre, presenta una baja variabilidad interanual, i.e. coeficientes de variación de $5,1 \%$ y $14,6 \%$ para $S$. bentincki y E. ringes, respectivamente. En tanto, la posición relativa del máximo

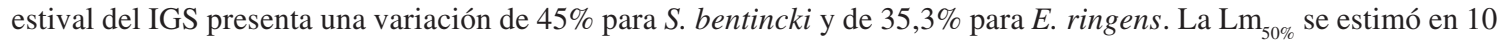
$\mathrm{cm}$ para $S$. bentincki y en $11,5 \mathrm{~cm}$ para E. ringens, la que es alcanzada en julio al término del primer año de vida en $S$. bentincki y en diciembre, al año y medio en E. ringens. Se postula que la mayor actividad reproductiva poblacional de ambas especies, centrada en agosto, es una respuesta adaptativa de las poblaciones al sistema de surgencia costera estacional de la zona centro-sur de Chile. La mayor productividad asociada al período de surgencias que comienza a mediados de septiembre y que se extiende hasta fines de marzo, sería favorable tanto para la sobrevivencia y crecimiento de estados larvales y juveniles como para el almacenamiento de energía de los adultos para ser utilizada en la reproducción durante el período invernal.

Palabras claves: época de desove, talla de primera madurez sexual, estrategia reproductiva, ecosistema de surgencia, peces pelágicos pequeños, Talcahuano (Chile).

\section{Reproductive period and mean size at first maturity for Strangomera bentincki and Engraulis ringens from 1993 to 1997 off central-southern Chile.}

\begin{abstract}
The reproductive period of Strangomera bentincki and Engraulis ringens and the size at first maturity $\left(\mathrm{Lm}_{50 \%}\right)$ are analyzed for the period 1993-1997. The reproductive indicators used are the gonadosomatic index (GSI) and the temporal frequency of macroscopic sexual maturity stages for mature and hydrated females. $\mathrm{Lm}_{50 \%} \mathrm{was}$ determined on the basis of $50 \%$ of mature females criteria using a logistic equation fitted to observed data. The month at maximum GSI, between July and September, has the lowest interannual variability, i.e. coefficients of variation of $5.1 \%$ y $14.6 \%$ for $S$. bentincki and $E$. ringens respectively. The month at secondary maximum GSI presented coefficients of variation of $45 \%$ for $S$. bentincki and of $35.3 \%$ for E. ringens. $\mathrm{Lm}_{50 \%}$ was estimated in $10 \mathrm{~cm}$ TL for S. bentincki and $11.5 \mathrm{~cm}$ for E. ringens. The $\mathrm{Lm}_{50 \%}$ of $S$. bentincki is achieved in July, at the end of the first year of life, while the $\mathrm{Lm}_{50 \%}$ of $E$. ringens is achieved in February, at about 1.5 year old. It is postulated that the populational reproductive period for both species, occurring in August during winter time, is a consequence of the adaptation to the seasonal coastal upwelling ecosystem off central-southern Chile. A higher productivity (e.g.food) in the coastal waters can be found as a consequence of the occurrence of upwelling events from middle September to late March. Under this hypothesis, larval stages and juveniles could find good conditions for growth and survival during spring and summer time, and adults could gain energy that could be used in gonad maturation for the reproduction during winter time, the less-productive season.
\end{abstract}

Key words: reproductive period, size at first maturity, reproductive strategy, small pelagic fish, upwelling ecosystem, Talcahuano (Chile). 


\section{INTRODUCCIÓN}

En Chile, Strangomera bentincki (Norman 1936) y Engraulis ringens (Jenyns 1842), conocidas localmente como sardina común y anchoveta, constituyen importantes recursos pesqueros y sustentan importantes pesquerías cuyo destino es la elaboración de harina, principalmente. En la zona centro-sur de Chile $\left(34^{\circ} \mathrm{S}-40^{\circ} \mathrm{S}\right)$, se desarrolla una importante actividad pesquera artesanal e industrial que se sustenta en la captura de estas especies, razón por la cual varios han sido los estudios biológicos y pesqueros que se han realizado (Aguayo y Soto, 1978; Serra, 1978; Arrizaga, 1981; Arrizaga y Veloso, 1982; Mujica y Rojas, 1984; Fonseca et al., 1986; Yáñez et al., 1990; 1992; Cubillos y Arancibia, 1993a,b,c; Orrego, 1993; Arancibia et al., 1994; Galleguillos et al., 1997; Cubillos et al., 1998a,b).

Desde el punto de vista de la biología reproductiva, ambas especies se caracterizan por ser desovantes parciales (Alheit et al., 1984; Santander et al., 1984; Yáñez, 1994), lo que implica que en un determinado período de tiempo es posible encontrar ejemplares en diferentes estados de madurez sexual (Arancibia et. al., 1994). Varios estudios indican que la máxima actividad reproductiva de $S$. bentincki ocurre entre julio y septiembre (Arrizaga, 1981; Cubillos y Arancibia, 1993a,b,c; Arancibia et al., 1994); y desde julio a diciembre en E. ringens (Cubillos y Arancibia, 1993a,b,c). A su vez, la talla de primera madurez sexual ha sido estimada en 11 $\mathrm{cm}$ de longitud total para S. bentincki por Arrizaga (1981) y Arancibia et al. (1994). En el caso de $E$. ringens la talla de primera madurez sexual fue estimada en $12 \mathrm{~cm}$ por Cubillos y Arancibia (1993a,b) sobre la base del máximo incremento porcentual del índice gonadosomático promedio por clases de tallas.

A pesar que los antecedentes relativos a la época y talla de primera madurez sexual parecen estar bien documentados, en este estudio se revisa los indicadores reproductivos con el objeto de analizar cambios en los patrones globales que se conocen de la actividad reproductiva de $S$. bentincki y $E$. ringens en un período de tiempo que abarca 5 años, desde 1993 a 1997. Los objetivos que se persiguen con esta revisión es analizar la estrategia reproductiva de S. bentincki y E. ringens en la zona centro-sur de Chile, particularmente en relación con la mayor productividad del medio asociada al proceso de surgencia costera, que en la zona centro-sur de Chile ocurre estacionalmente entre mediados de sep- tiembre hasta fines de marzo (Arcos y Navarro, 1986; Arcos, 1987). En este contexto, se analiza la estacionalidad del período reproductivo y sus cambios interanuales y se determina la talla de primera madurez sexual de ambas especies en la zona centro-sur de Chile.

\section{MATERIALES Y MÉTODOS}

\section{Fuente de la información}

La información básica proviene de muestreos biológicos específicos que realiza regularmente personal del Instituto de Investigación Pesquera en el marco de un programa de vigilancia biológico y pesquera de la pesquería de $E$. ringens y $S$. bentincki que se desarrolla en la zona centro-sur $\left(36^{\circ}-40^{\circ} \mathrm{S}\right)$, cuya pesca se desembarca principalmente en Talcahuano y Coronel. El diseño de muestreo para obtener indicadores biológicos específicos consiste en obtener muestras aleatorias semanales de la pesquería. Estas muestras son llevadas al laboratorio, donde se procede a medir cada ejemplar en su longitud y peso total, se extraen las gónadas y se clasifican por sexos, siendo las gónadas de las hembras pesadas con una balanza de precisión de 0,01 g. Además, cada ejemplar hembra se clasifica en un estado de madurez sexual utilizando una escala de madurez sexual de 5 puntos para desovantes parciales, adaptada de Holden y Raitt (1975) (Tabla 1).

\section{Epoca reproductiva}

La época reproductiva se determinó según la evolución mensual del índice gonadosomático promedio de las hembras. Se reconoce actividad reproductiva cuando estos índices alcanzan valores máximos dentro de un ciclo anual. El índice gonadosomático (IGS), se define según la expresión:

$$
I G S=\frac{P O}{P T-P O} 100
$$

donde PO es el peso del ovario (g) y PT el peso total del pez (g). El IGS promedio consiste en el promedio de los IGS de los individuos muestreados en cada mensuales. Para calcular el promedio se descartó las hembras hidratadas y los ejemplares menores a $10 \mathrm{~cm}$ de longitud total de ambos recursos. La señal anual del IGS en el período 1993-1997, se determinó a través de un promedio mensual de los IGS individuales. 
Con el objeto de analizar la persistencia interanual del momento en que ocurre el IGS máximo invernal, máximo secundario y mínimo otoñal en cada año, se ajustó un polinomio de $5^{\circ}$ a la secuencia temporal del IGS. En este análisis, los meses se numeraron de 1 a 12, considerando meses julianos (meses de 30 días) para definir el momento en que ocurre los IGS máximos dentro del ciclo anual.

El análisis de frecuencia de hembras maduras se realizó para cada año considerando tanto las hembras maduras como hidratadas, i.e. los estados de madurez sexual 3 y 4 . Con el objeto de analizar la señal estacional de los estados de madurez sexual, se acumuló la frecuencia de cada estado por mes para todo el período 1993-1997.

\section{Talla media de primera madurez sexual}

El análisis de la talla media de primera madurez sexual se determinó sobre la base del criterio del $50 \%$ de la fracción de hembras maduras (Arancibia et al., 1994), utilizando la siguiente ecuación logística:

2)

$$
P_{i}=\frac{1}{1+\exp (a-b L T)}
$$

donde $\mathrm{P}_{1}$ es la proporción de hembras sexualmente maduras a la longitud total (LT), a y b son constantes. El ajuste de la función de madurez se realizó mediante regresión no lineal. El error estándar de los parámetros se determinó sobre la base de estimadores asintóticos para la solución de mínimos cuadrados encontrada. La talla media de primera madurez sexual se estimó con información proveniente de los meses de julio - agosto del período 1993-97 en el caso de $S$. bentincki $(\mathrm{n}=1.354$, distribuidos en 19 clases de longitud) y entre julio y septiembre de igual período de tiempo en el caso de $E$. ringens $(\mathrm{n}=1.143$, distribuidos en 24 clases de longitud). La talla media de primera madurez sexual se determinó para todo el período de tiempo debido a que se está utilizando datos provenientes de un seguimiento de la actividad reproductiva cuyo muestreo no está diseñado, explícitamente, para tomar en cuenta el tamaño de los ejemplares. Así, solamente al incluir muestras de varios años se logra contar con un rango de tamaños que otorga contraste a la estimación de la ojiva de madurez.

Tabla 1. Escala empírica de madurez sexual para hembras de especies desovantes parciales (adaptada de Holden y Raitt, 1975).

Table 1. Empirical scale of sexual maturity for females of partial spawning fishes (after Holden and Raitt, 1975).

\begin{tabular}{|cll|}
\hline FASE & ESTADO & ASPECTO GENERAL \\
\hline I & $\begin{array}{l}\text { Inmaduro } \\
\text { traslúcidos, }\end{array}$ & $\begin{array}{l}\text { Ovarios ocupan cerca de 1/3 de la longitud de la cavidad abdominal, rosáceos, } \\
\text { ovocitos invisibles a simple vista. }\end{array}$ \\
II & $\begin{array}{l}\text { Virgen madurando } \\
\text { y en recuperación }\end{array}$ & $\begin{array}{l}\text { Ovarios ocupan casi la mitad de la longitud la cavidad abdominal. } \\
\text { Color rosado y ovocitos invisibles a simple vista. }\end{array}$ \\
III & Maduro & $\begin{array}{l}\text { Los ovarios cubren cerca de } 2 / 3 \text { de la cavidad abdominal. Color rosado a } \\
\text { amarillento. Aspecto granular, ovocitos visibles. Los ovocitos son grandes e } \\
\text { identificables a simple vista. Se observan vasos sanguíneos superficiales. }\end{array}$ \\
& Hidratado & $\begin{array}{l}\text { Los ovarios ocupan toda la cavidad abdominal. Color naranja opaco a rosado, } \\
\text { casi transparentes, con vasos sanguíneos superficiales poco visibles. Al } \\
\text { presionar fluyen ovocitos hidratados, transparentes, del doble de tamaño que } \\
\text { los ovocitos de una gónada madura. }\end{array}$ \\
& En regeneración & $\begin{array}{l}\text { Ovarios fláccidos, distendidos, contraídos a la mitad de la cavidad abdominal. } \\
\text { Las paredes son delgadas, flojas y lumen grande. Los ovarios } \\
\text { pueden contener aún restos de ovocitos opacos, maduros y en desintegración, } \\
\text { obscurecidos o translúcidos. Este ovario pasa a la etapa II de esta escala. }\end{array}$ \\
& &
\end{tabular}




\section{RESULTADOS}

\section{Epoca reproductiva}

La evolución mensual del índice gonadosomático (IGS) promedio de las hembras de S. bentincki y $E$. ringens, se presenta en la Fig. 1, observándose los cambios ocurridos en el período 1993-1997. En la serie de S. bentincki, 1993 se destaca por los altos valores de IGS observados y su cobertura temporal, el año 1994 por la presencia de un máximo secundario y los años 1996 y 1997 por los valores relativamente más bajos del IGS. Este último patrón también se verifica en E. ringens. En esta especie, los máximos secundarios son más persistentes y la evolución del IGS es muy similar dentro del ciclo anual en 1993 y 1994. La relación en fase de ambas series presenta un coeficiente de correlación (r) de 0,468 $(\mathrm{n}=55)$, mientras que la serie del IGS de anchoveta desfasada en un mes en relación con la de sardina, alcanza una correlación de 0,615 ( $\mathrm{n}=54)$, disminuyendo a una correlación de $0,555(\mathrm{n}=53)$ para un desfase de dos meses, y $r=0,394$ con un desfase de 3 meses.

EL IGS promedio por mes del período 199397, calculado a partir de todos los datos disponibles, indica que $S$. bentincki tiende a presentar máximos de IGS en agosto y $E$. ringens en septiembre. El IGS en $S$. bentincki se incrementa progresivamente desde febrero hasta agosto y disminuye más o menos abruptamente en octubre, para permanecer bajo entre noviembre y diciembre. En el caso de $E$. ringens, el IGS se incrementa progresivamente desde marzo-abril hasta septiembre, disminuye levemente entre octubre y noviembre para volver a incrementarse entre diciembre y enero (Fig. 2).

Para cada año del período 1993-1997, el índice gonadosomático (IGS) promedio de las hembras de $S$. bentincki y E. ringens se muestra en la Fig. 3, interpretándose su evolución mensual a través del ajuste de polinomios de $5^{\circ}$ a los datos. En $S$. bentincki, se observa que solamente en 1993 y 1994 el IGS tiende a presentar máximos secundarios entre enero y fines de marzo. A su vez, en la serie de tiempo se observa que los mayores valores del IGS tienden a presentarse entre julio y septiembre, aun cuando en 1996 y 1997 se observa los valores más bajos del IGS.

En el caso de E. ringens, se observa un patrón muy similar en el IGS durante 1993 y 1994. Sin embargo, a contar de 1995 se observa un cambio que se caracteriza porque la expresión de los máximos valores invernales del IGS tienden a adelantarse en el año, y se fortalece los máximos IGS estivales como ocurrió en enero de 1995, enero y febrero de 1996, y enero de 1997. Aunque, en 1997 nuevamente se observa un fortalecimiento de los máximos IGS invernales.

Los meses en que presentaron valores máximos del IGS estivales (enero-marzo), los valores mínimos en otoño (abril-junio) y los valores máximo
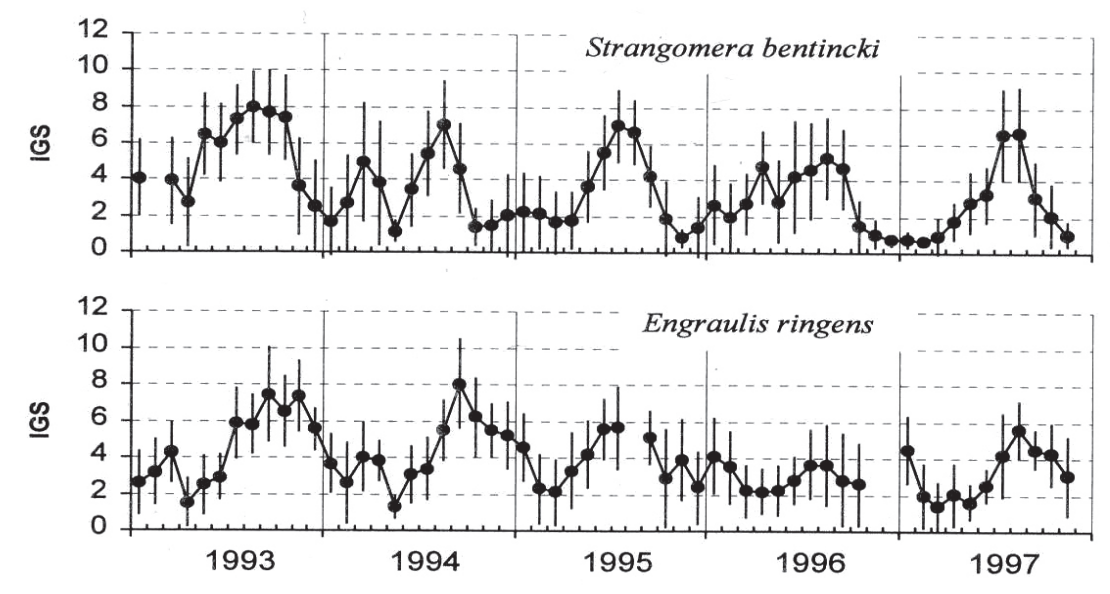

Figura 1. Cambios mensuales en el índice gonadosomático promedio de las hembras de S. bentincki y $\boldsymbol{E}$. ringens en el período 1993-1997. La barra vertical representa \pm 1 desviación estándar de los datos.

Figure 1. Monthly values of the gonadosomatic index (GSI) for females of S. bentincki and $E$. ringens between 1993 and 1997. The vertical bar represents \pm one standard deviation of the data. 

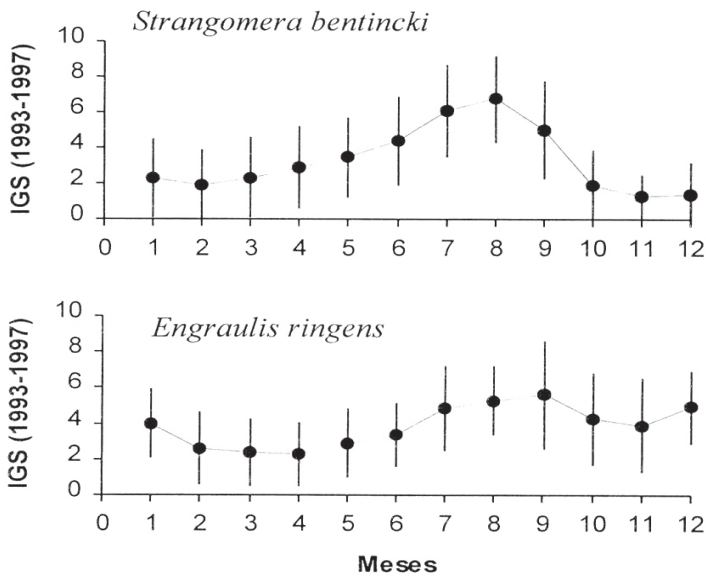

Figura 2. Promedio mensuales del IGS de $S$. bentincki y $E$. ringens para el período 1993-1997. La barra vertical representa \pm 1 desviación estándar de los datos.

Figure 2. Monthly average GSI of S. bentincki and E. ringens for the period 1993-1997. The vertical bar represents \pm one standard deviation of the data. invernales (julio-septiembre), interpolados de los modelos polinómicos ajustados, se resumen en la Tabla 2. Los valores máximos reportados en dicha tabla se refieren al máximo estimado a partir del modelo polinomial y no al valor del IGS máximo observado. En el caso de $S$. bentincki, se observa que el máximo IGS invernal presenta la más baja variabilidad interanual $(\mathrm{CV}=5,12 \%) \mathrm{y}$, en promedio, tiende a presentarse en agosto $(\mathrm{T}=7,65)$ con un IGS promedio de 6,33 (Tabla 2a). El entero del mes representa el término del mes, mientras que la fracción decimal los días dentro del mes siguiente. Así, en promedio el máximo IGS de $S$. bentincki tiende a ocurrir el 20 de agosto si se asume un mes de 30 días a contar del $1^{\circ}$ de enero.

La posición relativa del máximo estival dentro del año tiende a ocurrir en febrero, pero tiene una mayor variación ( $\mathrm{CV}=45,52 \%$, Tabla $2 \mathrm{a}$ ). Situación que se debe a que en $S$. bentincki en algunos años no se observa un máximo estival en el IGS, caso de 1995, 1996 y 1997 (Fig. 1, 3), situación que se refleja también en el valor promedio del IGS, i.e. 2,96 (Tabla 2a). En relación con los valores mínimos otoñales del IGS, éste tiende a presentarse en abril

Tabla 2. Mes (T) en que ocurrió el máximo IGS estival e invernal y el mínimo otoñal, interpolado de los ajustes de polinomios de $5^{\circ}$ a los datos mensuales observados del IGS.

Table 2. Month (T) at maximum GSI in summer (January-March) and winter time (July-September) and month at minimum GSI in fall time (April-June) as interpolated from the polynomies fitted to the monthly observed GSI.

\begin{tabular}{|c|c|c|c|c|c|c|}
\hline \multicolumn{7}{|c|}{ (a) Strangomera bentincki } \\
\hline & \multicolumn{2}{|c|}{ Máximo estival } & \multicolumn{2}{|c|}{ Mínimo otoñal } & \multicolumn{2}{|c|}{ Máximo invernal } \\
\hline año & $\mathrm{T}$ & IGS & $\mathrm{T}$ & IGS & $\mathrm{T}$ & IGS \\
\hline 1993 & 0,81 & 4,10 & 2,85 & 3,54 & 8,19 & 8,17 \\
\hline 1994 & 2,16 & 4,23 & 4,43 & 2,61 & 7,93 & 5,77 \\
\hline 1995 & 0,94 & 2,37 & 2,85 & 1,59 & 7,42 & 6,71 \\
\hline 1996 & - & - & - & - & 7,25 & 4,97 \\
\hline 1997 & 1,70 & 1,15 & 3,06 & 0,79 & 7,46 & 6,03 \\
\hline Promedio & 1,40 & 2,96 & 3,30 & 2,13 & 7,65 & 6,33 \\
\hline $\mathrm{CV}(\%)$ & 45,52 & 49,84 & 23,01 & 56,14 & 5,12 & 18,98 \\
\hline \multicolumn{7}{|c|}{ (b) Engraulis ringens } \\
\hline & \multicolumn{2}{|c|}{ Máximo estival } & \multicolumn{2}{|c|}{ Mínimo otoñal } & \multicolumn{2}{|c|}{ Máximo invernal } \\
\hline año & $\mathrm{T}$ & IGS & $\mathrm{T}$ & IGS & $\mathrm{T}$ & IGS \\
\hline 1993 & 1,96 & 3,73 & 4,48 & 2,33 & 9,60 & 7,40 \\
\hline 1994 & 1,84 & 4,05 & 4,70 & 2,30 & 9,72 & 6,74 \\
\hline 1995 & 1,00 & 4,62 & 2,46 & 1,94 & 6,97 & 5,63 \\
\hline 1996 & 1,06 & 4,14 & 4,04 & 1,93 & 7,46 & 3,64 \\
\hline 1997 & 1,00 & 4,33 & 3,54 & 1,21 & 8,62 & 4,99 \\
\hline Promedio & 1,37 & 4,18 & 3,84 & 1,94 & 8,47 & 5,68 \\
\hline CV (\%) & 35,28 & 7,96 & 23,24 & 23,24 & 14,59 & 26,05 \\
\hline
\end{tabular}



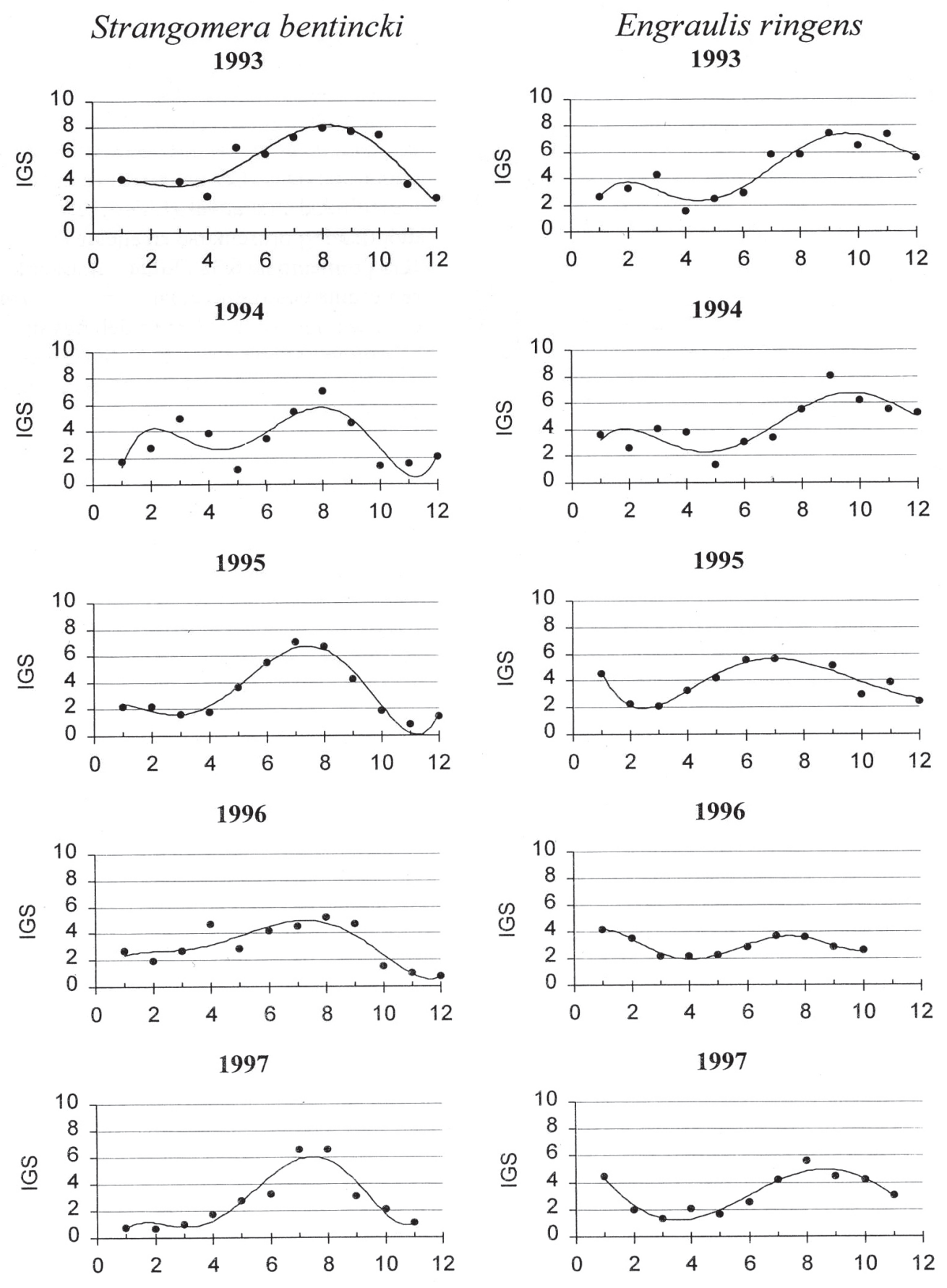

$\begin{array}{llllll}\mathbf{M} & \mathbf{E} & \mathbf{S} & \mathbf{E} & \mathbf{S}\end{array}$

Figura 3. Indice gonadosomático promedio mensual (IGS) de las hembras de S. bentincki y E. ringens en el período 1993-1997. La línea de tendencia fue determinada mediante ajuste de polinomios de cuarto y quinto grado.

Figure 3. Mean monthly gonadosomatic index (GSI) for females of S. bentincki and E. ringens between 1993 to 1997 . The tendency line was fitted by using polynomial models of $4^{\text {th }}$ and $5^{\text {th }}$ degree. 
$(\mathrm{T}=3,30)$ con un coeficiente de variación de $23,1 \%$ y un IGS promedio de 2,13 (Tabla 2a).

En el caso de E. ringens, también se observa una menor variabilidad interanual del mes en que ocurre el máximo IGS invernal, con una posición relativa centrada a mediados de septiembre $(T=8,47)$ y un coeficiente de variación de 14,59\%. El máximo estival tiende a presentarse en febrero $(\mathrm{T}=1,37)$, con una variación de $35,28 \%$, mientras que la posición relativa del mínimo otoñal ocurre en abril $(\mathrm{T}=3,84)$ con una variabilidad de sólo $25,8 \%$. El valor promedio del IGS máximo estival alcanza a 4,18, ligeramente inferior al máximo IGS promedio invernal de 5,68, pero el primero tiene una variación menor que el obtenido para el invierno (Tabla 2b).

En relación con la evolución mensual de la proporción de hembras maduras de S. bentincki, se observa que en 1993 se encontró más de un 50\% de hembras maduras entre mayo y octubre. En 1994 se encontró que entre julio y septiembre las hembras maduras se constituyeron con más del 50\%. En 1995, la proporción de hembras maduras sobrepasa el 50\% entre mayo y septiembre; mientras que en 1996, la proporción de hembras maduras presenta dos picos, uno en abril y otro en septiembre. A su vez, en 1997 se encuentra que entre abril y agosto las hembras maduras representan más del 50\%. En cuanto a $E$. ringens, se observa que la evolución temporal de la proporción de hembras maduras presenta rangos temporales más amplios que los registrados por el IGS, donde se observa un cambio en el patrón estacional del IGS a partir de 1995 (Fig. 4).

En la escala estacional, los datos acumulados para todo el período indican para $S$. bentincki que el estado de madurez sexual 2 (EMS 2), que representa ejemplares virginales en maduración, alcanza valores mayores al 60\% entre octubre y abril, mientras que su contribución es mínima en agosto. El EMS 3, que representa ejemplares maduros aporta con más del $60 \%$ entre mayo y septiembre, con un máximo en agosto que alcanza al $80 \%$. Los ejemplares hidratados (EMS 4), presenta la mayor con-

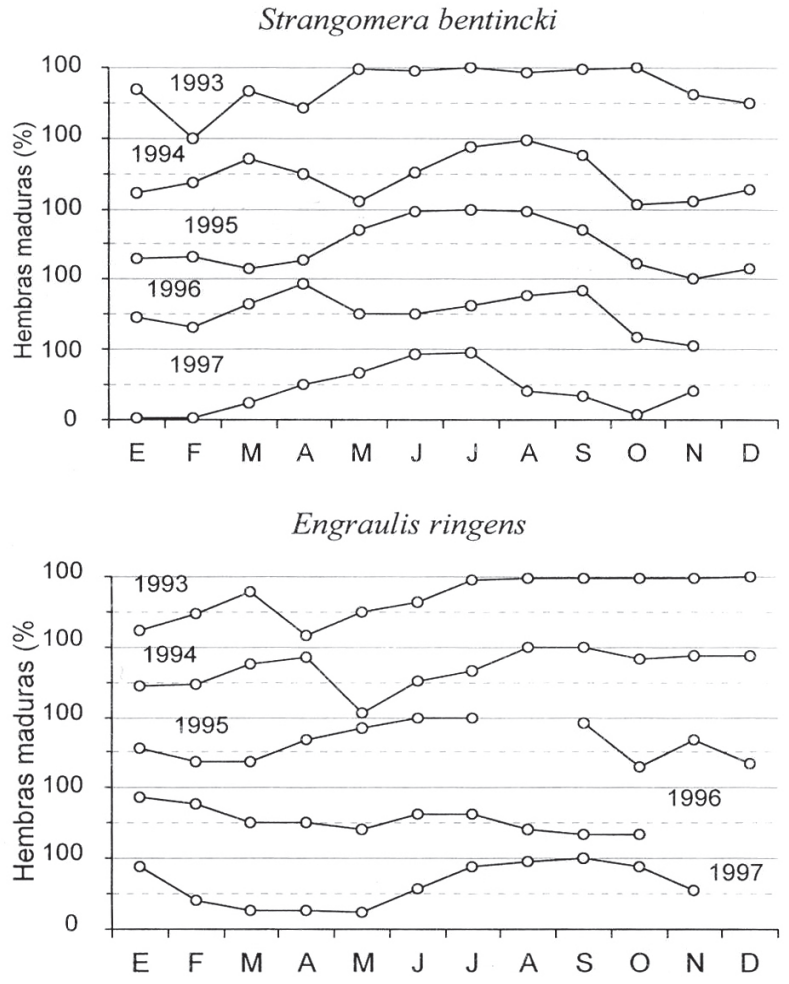

Figura 4. Proporción mensual de hembras maduras de $S$. bentincki y $E$. ringens entre 1993 y 1997. Estados de madurez sexual 3 and 4 (Tabla 1).

Figure 4. Monthly proportion of mature females of $S$. bentincki and E. ringens between 1993 and 1997. Sexual maturity stages 3 and 4 (see Table 1). 
tribución entre julio y agosto con cerca del $15 \%$, lo que marca la época de desove principal. Se destaca que la distribución temporal de estos ejemplares es menor al $1 \%$ entre noviembre y mayo. Por otra parte, los ejemplares que se encuentran en una etapa de regeneración (EMS 5), se presentan con mayor frecuencia entre octubre y diciembre disminuyendo a menos del $1 \%$ entre marzo y septiembre (Fig. 5).

En el caso de E. ringens, el EMS 2 presenta un máximo en abril y otros máximos relativos entre octubre y noviembre. El EMS 3, sigue la misma tendencia que la señal anual del IGS, observándose un incremento progresivo de hembras maduras desde abril hasta septiembre, interrupción entre octubre y diciembre, y un nuevo incremento entre diciembre y enero. El EMS 4, que representa hembras hidratadas, presenta valores máximos en julio y agosto, y también entre octubre y enero; mientras que el EMS 5 es máximo en enero, con alrededor del 16\% (Fig. 5).

\section{Talla media de primera madurez sexual}

La talla media de primera madurez sexual se estimó en 10 y $11,5 \mathrm{~cm}$ para $S$. bentincki y E. ringens, respectivamente (Fig. 6). Los parámetros de la ecuación logística estimados mediante mínimos cuadrados no lineales se resumen en la Tabla 3, observándose que son estadísticamente diferentes de cero al nivel del $95 \%$ de confianza y que el grado de ajuste es superior al $97 \%$.
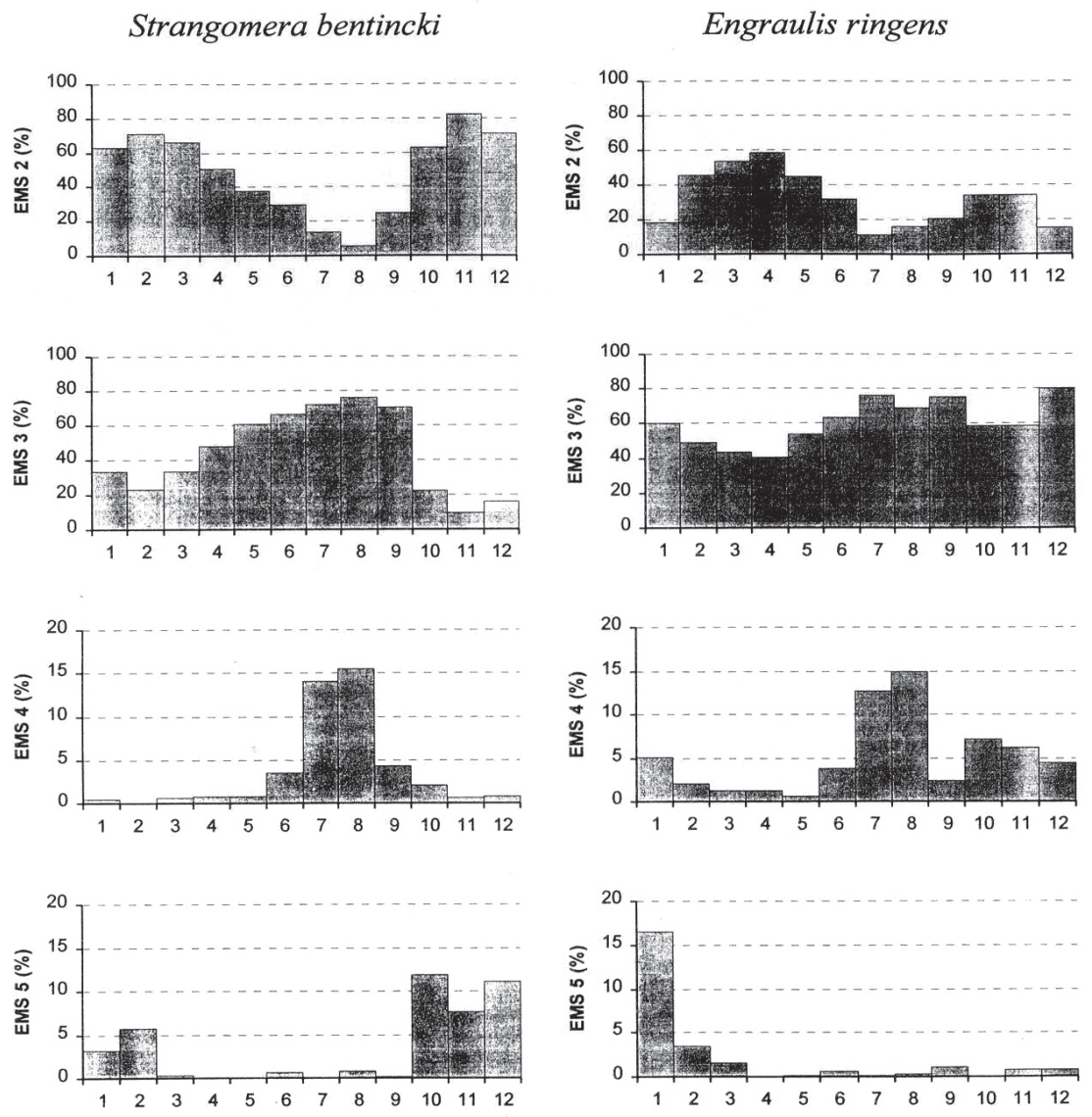

MESE S

Figura 5. Frecuencia (\%) mensual de los estados de madurez sexual de $S$. bentincki y $E$. ringens para todo el período de estudio.

Figure 5. Monthly frequency (\%) of sexual maturity stages of S. bentincki and $E$. ringens for the study period. 

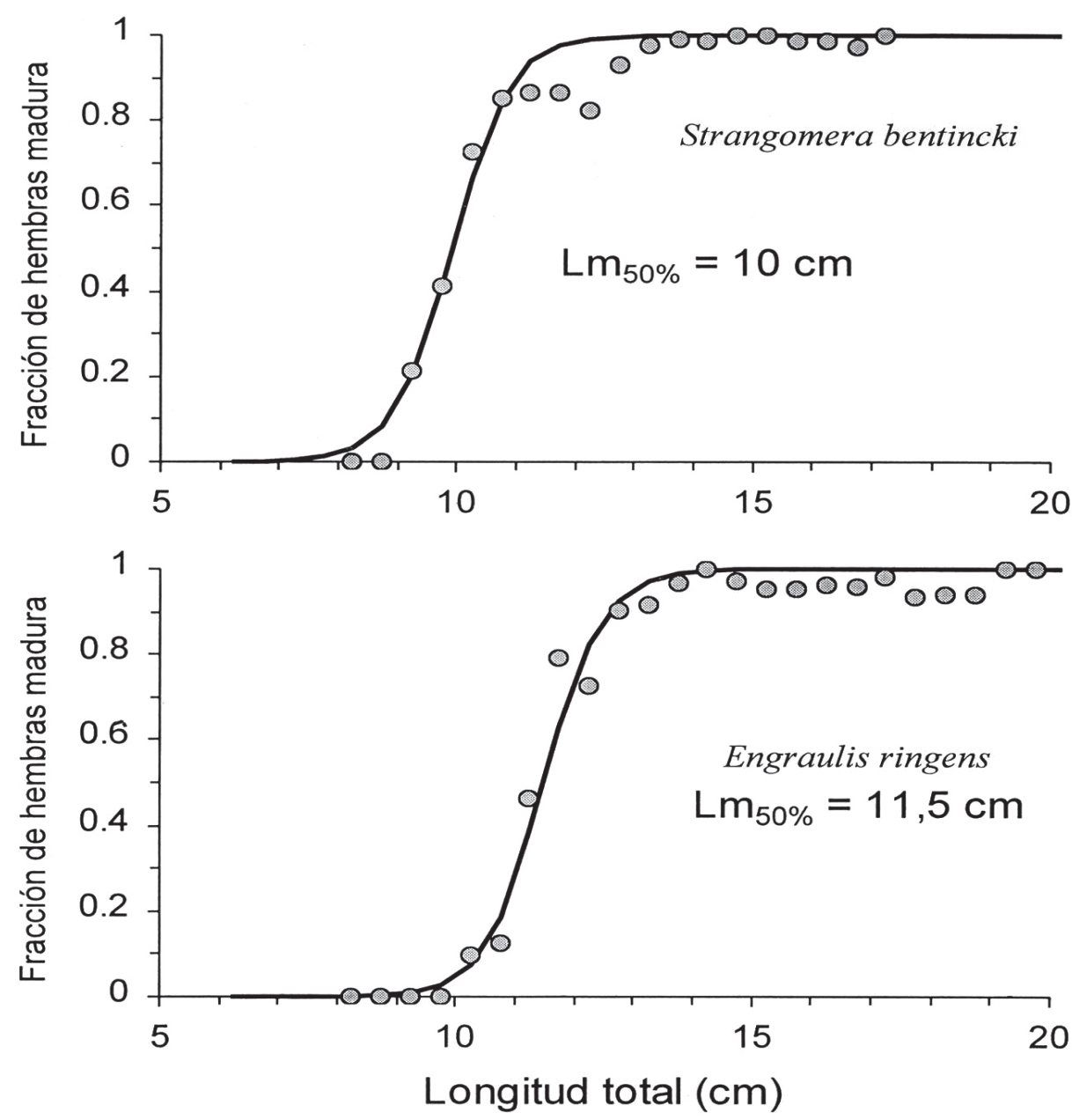

Figura 6. Talla de primera madurez sexual $\left(\mathrm{Lm}_{50 \%}\right)$ para las hembras de $S$. bentincki y E. ringens al nivel del $50 \%$ de hembras maduras. Zona centro-sur de Chile (ver Tabla 3).

Figure 6. Length at first sexual maturity $\left(\mathrm{Lm}_{50 \%}\right)$ for females of S. bentincki y $\mathrm{E}$. ringens by considering as criteria the proportion at $50 \%$ of maturity. Central-southern zone of Chile (see Table 3 )

Tabla 3. Resumen del ajuste del modelo logístico a la fracción de hembras maduras por clases de tallas. Entre paréntesis se indica el error estándar de los parámetros.

Table 3. Summary of the fitted logistic model to the proportion of mature females by length classes. The standard error is shown in parenthesis.

\begin{tabular}{|cccccc|}
\hline \multicolumn{2}{c}{ Parámetros } & & & \\
Especie & a & b & $\mathbf{r}^{\mathbf{2}}$ & $\mathbf{n}$ & P \\
\hline S. bentincki & $20,32(2,740)$ & $2,05(0,276)$ & 0,970 & 19 & $\mathrm{P}<0,05$ \\
E. ringens & $23,20(1,964)$ & $2,02(0,240)$ & 0,983 & 24 & $\mathrm{P}<0,05$ \\
\hline
\end{tabular}




\section{DISCUSIÓN}

Según Balbontín y Fischer (1981), una mayor fracción de los estados de madurez sexual «en maduración», «maduro»e «hidratado» refleja un aumento de la actividad ovárica, con el desarrollo de los ovocitos, y preparación de éstos previo al desove. En el caso de S. bentincki y E. ringens, la evolución temporal de la frecuencia de los estados de madurez 3 y 4 ha permitido corroborar las tendencias mostradas por el índice gonadosomático promedio. A su vez, la evolución temporal de las hembras hidratadas refleja la fracción de hembras desovantes diarias (Alheit et al., 1984), cuyo número de desoves se puede obtener a partir del área bajo la curva que se obtiene multiplicando la fracción de hembras hidratadas por el número de días calendario de cada mes. En julio y agosto, E. ringens alcanza una fracción de 12,6 y $14,9 \%$, respectivamente; mientras que para esos mismos meses fue de $14 \%$ y $15,6 \%$ en el caso de S. bentincki. En consecuencia, una hembra promedio de $E$. ringens y $S$. bentincki desovaría entre 4 y 5 veces en esos meses. Al año, el número de desoves alcanzaría a 13 en $S$. bentincki y 19 veces en $E$. ringens. Esta frecuencia del desove es similar a la informada para la anchoveta del norte (Engraulis mordax) por Hunter y Goldberg (1980) y para la anchoveta peruana por Alheit et al. (1984), Rojas y Oliva (1983) y Santander et al. (1984).

El momento en que ocurre el máximo IGS invernal presenta una escasa variación interanual en ambas especies y está centrado en agosto para $S$. bentincki y en septiembre para E. ringens. Este momento solamente expresa la máxima expresión del desarrollo gonadal de las hembras, produciéndose el desove con posterioridad. Sin embargo, la mayor frecuencia de hembras hidratadas está concentrada también en los meses de julio y agosto, razón por la cual, dado el carácter de desovantes parciales, entre julio y agosto deben estar ocurriendo desoves importantes. El momento en que se registra el máximo IGS invernal, según los polinomios ajustados, presenta la más baja variabilidad interanual, lo que se refleja en los coeficientes de variación encontrados, i.e. $5,12 \%$ y $14,6 \%$ para $S$. bentincki y E. ringens, respectivamente. En cambio, la posición relativa de los máximos IGS estivales tienden a ser más variables, con coeficientes de variación mayores al $35 \%$.

Esta mayor variación en el momento en que ocurre actividad reproductiva en el verano (enero-marzo) se puede deber al efecto individual, más que al poblacional, especialmente en el caso de S. bentincki. Es decir, en la época estival y bajo ciertas condiciones ambientales es probable que se produzcan desoves, dado que es un desovador parcial (Yáñez, 1994). Sin embargo, la época de máxima actividad reproductiva hacia el término del invierno es más consistente y menos variable interanualmente, proceso que debería estar relacionado con el efecto poblacional. En E. ringens, sin embargo, los máximos IGS estivales son más persistentes y pueden llegar a ser máximos dentro del ciclo anual, situación que podría ser explicada por los individuos que alcanzan la talla de primera madurez sexual y que provienen del desove invernal.

En E. ringens, la talla media de primera madurez sexual estimada es inferior en $0,5 \mathrm{~cm}$ a la informada anteriormente en $12 \mathrm{~cm}$ LT por Cubillos y Arancibia (1993b), usando el criterio del máximo incremento porcentual del IGS entre clases de tallas consecutivas (Finucane y Collins, 1984). De acuerdo con la curva de crecimiento de E. ringens (Cubillos et al., 1998b), la talla media de primera madurez sexual es alcanzada entre enero y febrero, al año y medio de vida aproximadamente tomando en cuenta el primero de julio como fecha de cumpleaños arbitraria. Esta dinámica asociada al crecimiento podría explicar el incremento en la actividad reproductiva de $E$. ringens entre diciembre y febrero, sugiriéndose con esto que la actividad reproductiva estival de esta especie se debe a los ejemplares que se reproducen por primera vez.

La talla media de primera madurez sexual, estimada en este estudio con información de todo el período 1993-97 para los meses de julio y agosto, es un centímetro inferior a la comunicada para $S$. bentincki por otros autores (e.g. Arrizaga, 1981; Cubillos y Arancibia, 1993b; Arancibia et al., 1994), situación que probablemente se debe al hecho de integrar varios años en la estimación. En todo caso, de acuerdo con el crecimiento de $S$. bentincki, los $10 \mathrm{~cm}$ LT se alcanza exactamente entre julio y agosto, al término del primer año de vida (Cubillos y Arancibia, 1993a, Cubillos et al., 1998).

A pesar que las tendencias promedio solamente han sido calculadas con 5 años de información, se considera que la mayor actividad reproductiva entre julio y agosto en $S$. bentincki y entre julio y septiembre en E. ringens podría ser consecuencia de un adaptación de la reproducción al medio. ¿Qué tan representativo puede ser el corto período de tiempo observado en relación con una escala de tiempo 
evolutiva? Responder esta pregunta es un desafío enorme y probablemente no podrá nunca ser contestada. Sin embargo, los primeros antecedentes relativos a la época reproductiva de $S$. bentincki fueron aportados por Brandhorst \& Rojas (1965, fide Serra et al., 1979), quienes sugieren que la época de desove ocurre entre junio y septiembre; Arrizaga (1981), señala también a estos meses como la época de desove más intensa entre 1965 y 1973. En la década de los 90, Cubillos y Arancibia (1993b,c) comunica máximos valores de IGS en agosto para 1990 y 1991; similar resultado informa Arancibia et al. (1994) para 1992, períodos que no fueron considerados en este estudio. En este contexto, en un período que abarca desde 1965 hasta 1997 (i.e. más de 30 años), la época de desove de $S$. bentincki es consistente y está referida siempre a los meses de invierno (i.e. julio-agosto), situación que fortalece y apoya la tesis de significancia adaptativa de la época de mayor actividad reproductiva.

De acuerdo con Bakun y Parrish (1982, 1990), Parrish et al. (1983) y Bakun (1996), en especies pelágicas costeras como sardinas y anchovetas, la reproducción ocurre en épocas y áreas donde la turbulencia y el transporte fuera de la costa son bajos. A su vez, Roy et al. (1992) sugiere para peces pelágicos costeros del oeste de Africa que diferencias en los meses de máxima actividad reproductiva se encuentran próximos al valor de $5-6 \mathrm{~m} \cdot \mathrm{s}^{-1}$ de la velocidad del viento, valor representativo de la hipótesis de «ventana ambiental óptima» de Cury \& Roy (1989).

Si la época de mayor actividad reproductiva está bien definida para los meses de julio-agosto en $S$. bentincki y julio-septiembre en E. ringens, y la mayor productividad en la región ocurre en la época estival, ¿cómo estas especies establecen su presupuesto energético si la reproducción comienza en la estación menos productiva en la zona centro-sur? En S. bentincki, Arrizaga (1981), analizó el ciclo anual de contenido de grasa entre 1966 y 1967, observándose máximos entre diciembre y abril y mínimos entre septiembre y octubre. En consecuencia, es probable que la energía disponible durante la época de máxima producción plantónica no es utilizada inmediatamente para la reproducción, sino más bien destinada al crecimiento (particularmente en juveniles) y también almacenada para ser utilizada en la producción de gametos durante la época invernal.
Por otra parte, Cubillos y Arancibia (1993c) y Cubillos et al. (1998b) encuentran que la tasa de crecimiento de estos pelágicos exhibe cambios estacionales en la tasa de crecimiento, donde la más baja tasa de crecimiento ocurre en invierno. Así, los individuos de ambas especies podrían estar aprovechando el período de surgencia costera, que se extiende desde mediados de septiembre hasta marzo (Arcos y Navarro, 1986; Arcos, 1987), para el crecimiento y la engorda; y la época invernal para canalizar energía a la producción de gamentos. Poblacionalmente, la producción de huevos en invierno estaría sincronizada con el inicio del período de surgencias en primavera (fines de septiembre). Así los estados larvales podrían encontrar alimento necesario para asegurar el crecimiento y la sobrevivencia (Cushing, 1975, 1990).

Sin embargo, aunque la estrategia reproductiva satisface la dinámica de $S$. bentincki, el máximo IGS estival de E. ringens no podría ser una consecuencia de esta estrategia. La hipótesis que la máxima actividad reproductiva de $E$. ringens se sustenta en los individuos de $11 \mathrm{~cm}$ que están en condiciones de reproducirse por primera entre enero y febrero, podría explicar el comportamiento del IGS de esta especie. En efecto, en peces la maduración de las gónadas requiere una gran cantidad de energía, de tal manera que usualmente el desove ocurre después del período con condiciones más favorables para la alimentación (Wootton, 1992). Debido al crecimiento más lento de E. ringens (Cubillos et al., 1998b), al término del primer año de vida los juveniles alcanzan entre 8 y $9 \mathrm{~cm}$ en julio-agosto y $11 \mathrm{~cm}$ en diciembre, de tal manera que entre septiembre y diciembre (época de surgencia), los juveniles de $E$. ringens se alimentarían para que una fracción lleve a cabo su primera reproducción a partir de diciembre-enero.

Es probable que la extensión del período de desove en cada año sea dependiente de las condiciones ambientales y de la estructura demográfica del stock. En este contexto, es altamente necesario comenzar a investigar la influencia de factores bióticos y abióticos sobre el ciclo reproductivo de estos pelágicos pequeños, no sólo en lo que respecta a la ocurrencia de actividad ovárica sino también sobre los efectos en la extensión e intensidad del período reproductivo. 


\section{AGRADECIMIENTOS}

Parte de los resultados de este estudio fueron obtenidos en el marco del proyectos FIP $\mathrm{N}^{\circ}$ 94-38 «Reproducción y reclutamiento de sardina común y anchoveta en la zona centro-sur» y FIP $\mathrm{N}^{\circ}$ 96-10 «Evaluación indirecta del stock de anchoveta y sardina común en la zona centro-sur». Los autores agradecen al Consejo de Investigación Pesquera las facilidades otorgadas para publicar parte de los resultados de dichos estudios. También se agradece a los tres revisores del manuscrito, quienes aportaron con valiosos comentarios y sugerencias.

\section{REFERENCIAS}

Aguayo, H.M. y S.B. Soto. 1978. Edad y crecimiento de la sardina común (Clupea (Strangomera) bentincki) en Coquimbo y Talcahuano. Invest. Pesq. (Chile), 27: 1-55.

Alheit, J., V.H. Alarcón y B.J. Macewics. 1984. Spawning frequency and sex ratio in the Peruvian anchovy, Engraulis ringens. Calif. Coop. Oceanic Fish. Invest. Rep. 25: 43-52.

Arancibia, H., L. Cubillos, J. Remmaggi y R. Alarcón. 1994. Determinación de la talla de primera madurez sexual y fecundidad parcial en la sardina común, Strangomera bentincki (Norman, 1936), del área de Talcahuano, Chile. Biol. Pesq. 23: $11-17$

Arcos, D. 1987. Seasonal and short time scale variability in copepod abundance and species composition in an upwelling area off Concepción coast, Chile. Ph.D. Thesis Dissertation, State University of New York, Stony Brook, 203 pp.

Arcos, D.F. y N. Navarro. 1986. Análisis de un índice de surgencia para la zona de Talcahuano, Chile (Lat. $37^{\circ} \mathrm{S}$ ). Invest. Pesq. (Chile), 33: 91-98.

Arrizaga, A. 1981. Nuevos antecedentes biológicos para la sardina común, Clupea (Strangomera) bentincki Norman 1936. Bol. Soc. Biol. Concepción, 52: 5-66.

Arrizaga, A. y C. Veloso. 1982. Estimación de mortalidades (M, F y Z) y del coeficiente de capturabilidad (q) en la sardina común, Clupea (Strangomera) bentincki Norman 1936, de Talcahuano-Chile. Monogr. Biol., 2: 39-49.
Bakun, A. y R.H. Parrish. 1982. Turbulence, transport, and pelagic fish in the California and Peru current systems. Rep. Calif. coop. oceanic Fish. Invest., 123: 99-112.

Bakun, A. y R.H. Parrish. 1990. Comparative studies of coastal pelagic fish reproductive habitats: the Brasilian sardine (Sardinella aurita). J. Cons. int. Explor. Mer, 46: 269-283.

Bakun, A. 1996. Patterns in the ocean: ocean processes and marine population dynamics. University of California Sea Grant, San Diego, California, USA, and Centro de Investigaciones Biológicas de Noroeste, La Paz, Baja California Sur, México, 323 pp.

Balbontín, F. y W. Fisher. 1981. Ciclo sexual y fecundidad de la merluza Merluccius gayi, en la costa de Chile. Rev. Biol. Mar. 17(3): 285-334.

Cubillos, L. y H. Arancibia. 1993a. Análisis de la pesquería de sardina común y anchoveta del área de Talcahuano, situación actual y perspectivas. Documentos Técnicos del Instituto de Investigación Pesquera (IIP), Talcahuano, Chile 2(2): 1-19.

Cubillos, L. y H. Arancibia. 1993b. Análisis de la pesquería de sardina común (Strangomera bentincki) y anchoveta (Engraulis ringens) del área de Talcahuano, Chile. Invest. Mar., Valparaíso, 21: 3-21.

Cubillos, L. y H. Arancibia. 1993c. On the seasonal growth of common sardine (Strangomera bentincki) and anchovy (Engraulis ringens) off Talcahuano, Chile. Rev. Biol. Mar., 28(1): 43-49.

Cubillos, L., M. Canales, A. Hernández; D. Bucarey, L. Vilugrón y L. Miranda. 1998a. Poder de pesca, esfuerzo de pesca y cambios estacionales e interanuales en la abundancia relativa de Strangomera bentincki y Engraulis ringens en el área frente a Talcahuano, Chile (1990-1997). Invest. Mar., Valparaíso, 26: 3-14.

Cubillos, L., R. Alarcón, D. Bucarey, M. Canales, P. Sobarzo y L. Vilugrón. 1998b. Evaluación indirecta del stock de anchoveta y sardina común en la zona centro-sur. Informes Técnicos FIP, FIP-IT/ 96-10, 223 pp.

Cury, P. y C. Roy. 1989. Optimal environmental window and pelagic fish recruitment success in upwelling areas. Can. J. Fish. Aquat. Sci. 46: 670680. 
Cushing, D.H. 1975. Marine ecology and fisheries. Cambridge University Press, Cambridge. 275 pp.

Cushing, D.H. 1990. Plankton production and yearclass strength in fish populations: an update of the match/mismatch hypothesis. Adv. Mar. Biol., 26: 249-293.

Finucane, J.H. y L.A. Collins. 1984. Reproductive biology of cero, Scomberomorus regalis, from the coastal waters of south Florida. Northeast Gulf Sci., 7(1): 101-107.

Fonseca, T.R., E. Yáñez y O. Barra. 1986. Relación entre la temperatura superficial del mar y capturas comerciales en el área de Talcahuano entre 1965 y 1976. In: P. Arana (ed.) La Pesca en Chile, Escuela de Ciencias del Mar, UCV, Valparaíso, pp. 243-248.

Galleguillos, R., L. Troncoso, J. Monsalves y C. Oyarzún. 1997. Diferenciación poblacional en la sardina chilena Strangomera bentincki (Pisces: Clupeidae): análisis genético de variabilidad proteínica. Rev. Chil. de Hist. Nat., 70: 351-361.

Holden, M.J. y D.F.S. Raitt. 1975. Manual de ciencia pesquera. Parte 2: Métodos para investigar los recursos y su aplicación. Doc. Téc. FAO. Pesca (115), Rev. 1: 211 pp.

Hunter, J.R. y S. Goldberg. 1980. Spawning incidence and batch fecundity in the northern anchovy, Engraulis mordax. Fish. Bull., 77(3): 641652.

Mujica, A. y O. Rojas. 1984. Fecundidad y estructura poblacional de sardina común (Clupea bentincki, Norman) (Clupeiformes-Clupeidae). Invest. Pesq. (Chile), 31: 59-69.

Orrego, A.H. 1993. Edad y crecimiento de la sardina común Strangomera bentincki (Norman, 1936) (Pisces: Clupeidae) a través de los microincrementos en los otolitos sagittae. Tesis de Magister en Ciencias, Escuela de Graduados, Universidad de Concepción. 84 pp.

Parrish, R.H., A. Bakun, D.M. Husby y C.S. Nelson. 1983. Comparative climatology of selected environmental processes in relation to eastern boundary current pelagic fish reproduction. In: G.D. Sharp and J. Csirke (eds.). Proceedings of the Expert Consultation to Examine Changes in Abundance and Species Composition of Neritic Fish Resources, San José, Costa Rica, April 1983. FAO Fish. Rep. 291(3): 731-777.
Rojas, O. y J. Oliva. 1993. Evaluación del stock desovante de anchoveta de la zona norte por el método de producción de huevos. Programa evaluación directa de recursos pelágicos de la zona norte. IFOP (Chile), 37 pp. + anexos.

Roy, C., P. Cury y S. Kifani. 1992. Pelagic fish recruitment success and reproductive strategy in upwelling areas: environmental compromises. In: A.I. Payne, L. Brink, K.H. Mann and R. Hilborn (eds.). Benguela Trophic Functioning, S. Afr. J. mar. Sci., 12: 135-146.

Santander, H., J. Alheit y P.E. Smith. 1984. Estimación de la biomasa desovante de anchoveta peruana, Engraulis ringens, en 1981 por aplicación del Método de Producción de Huevos. Bol. Inst. Mar Perú, Callao, 8: 209-250.

Serra, J.R. 1978. La pesquería de sardina común (Clupea (Strangomera) bentincki) y anchoveta (Engraulis ringens) de Talcahuano: análisis de su desarrollo y situación actual. Invest. Pesq. (Chile), 29: 1-21.

Serra, J.R., O. Rojas, M. Aguayo, F. Inostroza y J. Cañón. 1979. Sardina común. In: Estado actual de las principales pesquerías nacionales. Bases para un desarrollo pesquero. Peces I. CORFO-IFOP, 36 pp.

Yáñez, E., M.A. Barbieri y A. Montecinos. 1990. Relaciones entre las variaciones del medio ambiente y las fluctuaciones de los principales recursos pelágicos explotados en la zona de Talcahuano, Chile. In: M.A. Barbieri (ed.). Perspectivas de la Actividad Pesquera en Chile, Escuela de Ciencias del Mar, UCV, Valparaíso, pp. 49-62.

Yáñez, E., M.A. Barbieri y L. Santillán. 1992. Longterm environmental variability and pelagic fisheries in Talcahuano, Chile. In: A.I. Payne, L. Brink, K.H. Mann and R. Hilborn (eds.). Benguela Trophic Functioning, S. Afr. J. mar. Sci. 12: 175-188.

Yáñez, R.A. 1994. Determinación del estado de madurez sexual de la sardina común Strangomera bentincki (Norman, 1936). Seminario Biología Marina, Facultad de Ciencia, Universidad Católica de la Santísima Concepción, Concepción, 28 p.

Wooton, R.J. 1992. Ecology of teleost fishes. $2^{\circ} \mathrm{Ed}$. Chapman \& Hall, Londres. 